ciones que el obispo de Astorga tenía con la corte papal y con la iglesia de Santiago de los Españoles en Roma, que pudieron haberle recomendado personalmente las habilidades escultóricas del andaluz ${ }^{38}$. Estos son los factores que contribuyeron, sin lugar a dudas, a aclamar la victoria de Becerra en el concurso escultórico del retablo mayor de la Catedral de Astorga a mediados del siglo XVI.

CARMEN FRACCHIA

Universidad de Londres

\title{
RELACIONES ENTRE EL PINTOR ALONSO SÁNCHEZ COELLO Y LA FAMILIA FARNESIO
}

Aunque el pintor valenciano Alonso Sánchez Coello cultivó otros géneros pictóricos como el religioso, su fama proviene de su faceta como retractista de la casa real. Tras una primera formación en la corte portuguesa y en Flandes junto a su maestro Antonio Moro, le encontramos, desde 1555, al servicio de Felipe II para quien realizó múltiples encargos hasta su muerte, el 8 de agosto de 1588. Pacheco nos relató los numerosos favores con que le obsequiaba Felipe II, quien, de creer al tratadista, visitaría asiduamente su taller como otrora hiciera Alejandro con Apeles, pero también su arte sería admirado a nivel internacional por personajes de la talla de «los papas Gregorio XIII y Sixto V, los grandes duques de Florencia y de Saboya y el cardenal Alejandro Farnesio, hermano del Duque de Parma» ${ }^{1}$. Hasta que R. M. Mulcahy no dio a conocerr siete cartas de la correspondencia entre el pintor y el cardenal Farnesio ${ }^{2}$ y C. Robertson las gestiones de este último para conseguir una vacante para el hijo del pintor ${ }^{3}$, no se pudieron demostrar documentalmente estas relaciones artísticas materializadas en varios retratos.

Todos los indicios apuntan a que el primer miembro de la familia Farnesio con el que Sánchez Coello mantiene contactos es con el joven Príncipe de Parma y Piacenza, Alejandro, a quien retrata en $1561^{4}$ cuando se encontraba en la corte de su tío Felipe II. El fin de esta obra sería el de integrarse en una galería de retratos o quizá ser enviada a los Duques de Parma, los progenitores del Príncipe, para informarles del óptimo desarrollo de éste ${ }^{5}$ y atenuar las sospechas farnesianas que vieron los designios ${ }^{6}$ de Felipe II casi como un «secuestro». Margarita de

38 Ver nota anterior.

1 Pacheco, F., Arte de la Pintura, ed. a cargo de J. Sánchez Cantón, Madrid, 1956, pp. 146 y 147.

2 Mulcahy, R. M., «Alonso Sánchez Coello and Cardinal Alessandro Farnesse», Burlington Magazine, mayo 1992, pp. 305-308.

${ }^{3}$ Robertson, C., «Il Gran cardinale», Alessandro Farnese, Patron of Arts, New Haven, 1992.

${ }^{4}$ Este retrato pertenece a la Algur H. Meadows Collection. Meadows Museum, Southern Methodist University, Dallas, Texas. Cat. Alonso Sánchez Coello y el retrato en la corte de Felipe II, Madrid, 1990, p. 147, n. ${ }^{\circ} 30$. Se conserva una copia de medio cuerpo en la Galleria Nazionale de Parma. M Kusche en su estudio «Sofonisba e il ritratto di rappresentanza ufficiale nella corte spagnola», en el catálogo Sofonisba Anguissola e le sue sorelle, Cremona, 1994, p. 133, se lo atribuye a Antonio Moro, pero se debe tener en cuenta que Moro abandona la corte española en 1560.

5 Este fue el destino del retrato del Príncipe realizado por Antonio Moro en 1557. Sobre este tema, véase Meijer, B., Parma e Bruxelles. Committenza e collezionismo farnesiani alle due corti. Parma, 1988, pp. 120-125.

${ }^{6}$ Una de las cláusulas del tratado secreto de Gand, firmado entre Felipe II y los Farnesio en 1556, era la de enviar a la corte de Felipe II al joven heredero de Ottavio Farnesio para que fuese allí educado. De esta manera, Felipe II se aseguraría la fidelidad de los Farnesio y pondría fin a las veleidades de esta familia con Francia. Para profundizar en este tema, resulta fundamental la obra de Drei, G., I Farnese. Grandezza e decadenza di una dinastia italiana, Roma, 1954, caps. VIII y IX. 
Austria no sólo se preocupa por obtener retratos de su hijo, sino que, mientras es gobernadora de los Países Bajos, llegan a sus manos desde la corte madrileña otros retratos de la familia real. Así, en 1563, hace copiar al maestro Givanino ${ }^{7}$ un retrato de su hijo Alejandro y otro del rey Felipe II para enviarlos a Parma. La primera noticia que tenemos de estos dos cuadros provenientes de España y después copiados, la encontramos en una carta que escribe desde Bruselas, el 20 de marzo de 1563, el guardarrobba de la duquesa de Parma - Giovanni Aligrandi- a Paolo Viteli, hombre de confianza de Margarita. En ella leemos: «il ritrato di sua maestà [Felipe II] è comincciato e di qua da pascua sara finito si dara poi principio a fare quelo del $s^{o r}$ principe nos-

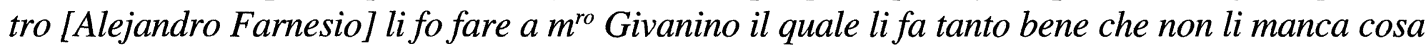
alcuna» ${ }^{8}$. En otra carta de Francesco Landi a P. Viteli, fechada en la capital belga el 25 de abril de 1563, aparece la siguiente postdata: «Ms. Giovanni guardarobba baccia le mani di v.s.Ill ${ }^{\text {ma }} e$ dice (...) chel ritratto del Re e finito et e bellisimo e molto conforme a quel venuto di Spagna: $E$ fra 2 giorni darà principio al ritrato del $s^{\text {or }}$ principe y come saranno finiti li inviera in italia» 9. El 3 de mayo será el propio G. Aligrande quien escriba a P. Vitelli para informarle personalmente: «Credo v.s.Ill ${ }^{m a}$ avera saputo come $m^{\text {ro }}$ givanino pintore a finito il ritrato di sua maesta il quale è benissimo fatto et contenta molto a sua ecc a fra doi giorni si dara principio a far quelo del sor principe e poi con la prima comodita li mandero a v.s. ill ${ }^{m a}{ }_{\gg}{ }^{10}$. No sería una hipótesis a descartar que los retratos enviados desde España fuesen de Sánchez Coello o de su círculo, pues es el único - junto a Sofonisba Anguissola, de aceptar que ella realizase el retrato del Príncipe conservado en la Galería Nacional de Irlanda ${ }^{11}$ - que retrata a Alejandro Farnesio durante su estancia en España.

En 1572, una vez finalizada su primera etapa como gobernadora, solicitó, por medio de Pietro Aldobrandini - uno de los agentes de la familia Farnesio-, el permiso de su hermanastro para que Sánchez Coello retratase a sus sobrinas Isabel Clara Eugenia y Catalina Micaela (Apéndice Documental, I). El rey Felipe no accede a esta petición, al menos por el momento, «por estar la mayor dellas tan descolorida y malita como esta no le pareçe ser este tiempo a proposito, que llegado ay y aviendo mejorado la niña, se lo torne a acordar, que el dira la forma que se terna para averlas de rretratar y ynbiar los etratos a la duquesa». Esta preocupación del rey porque la imagen de su familia sea lo más acorde con la idea de «majestad» que pretendían transmitir estas obras, se manifiesta en otras ocasiones en que no juzga conveniente retratar a sus hijos por estar enfermos ${ }^{12}$. El principal problema que plantea este retrato, si se llegó a realizar, es el de su identificación. De lo que no existen dudas es de que guardaría una enorme similitud con los retratos dobles de las infantas conservados en el Museo del Prado y en la colección de la reina de Inglaterra, puesto que, en 1572, las infantas contarían con cinco y cuatro años de edad ${ }^{13}$. El que el retrato se debiese realizar «del natural»,

\footnotetext{
${ }^{7}$ En la documentación consultada en el A.S.Pr., no hemos encontrado ninguna referencia a este maestro.

8 A.S.Pr., Carteggio Farnesiano, Estero, Paessi Bassi, b.108.

9 A.S.Pr., Ibidem.

${ }^{10}$ A.S.Pr., Ibidem.

${ }^{11}$ Este retrato, atribuido en un primer momento a Sánchez Coello y después tenido como anónimo, es adjudicado a la pintora cremonense por M. Kusche en su artículo «Sofonisba Anguissola en España, retratista en la corte de Felipe II junto a Alonso Sánchez Coello y Jorge de la Rua», en A.E.A., 1989, p. 404. Véase también la ficha n. ${ }^{\circ} 25$ del cat. Sofonisba..., 1994, donde se identifica como Alejandro Farnesio al joven desconocido del Museo Nacional de Arte Antica de Lisboa. El retrato de Sofonisba, pintado alrededor de 1561, o una copia del mismo, se encontraría en Roma antes de 1566, puesto que se emplea en uno de los frescos que pinta Taddeo Zuccaro en la sala de los Fasti Farnesiani de la villa de Caprarola.

12 Serrera, J. M., «Alonso Sánchez Coello y la mecánica del retrato de corte», cat. Alonso Sánchez Coello y el retrato en la corte de Felipe II, Madrid, 1990, pp. 38-63.

${ }^{13}$ El retrato del Museo del Prado (n. ${ }^{\circ}$ inv. 1138) en la ficha n. 22 del cat. Alonso Sánchez Coello..., 1990, p. 143, es fechado por S. Breuer-Hermann hacia 1575 atendiendo a la edad de las infantas y al vestuario. Sin embargo, Carmen Garrido en la misma obra (pp. 218 y 219), estima más plausible datar el retrato hacia 1570 considerando que su edad rondaría los
} 
hace pensar que, en este momento, no se disponía de un retrato de las infantas del que sacar una réplica.

Aparte de estos contactos con la rama ducal de la familia Farnesio, Sánchez Coello mantuvo durante años una fluida correspondencia — desde 1579 a 1588, año de su muerte - con el cardenal Alessandro Farnesio, uno de los mecenas artísticos de mayor relevancia en la Roma de la segunda mitad del siglo XVI ${ }^{14}$. No está muy claro cómo surge la relación entre ambos ${ }^{15}$, pero el pintor recurre al cardenal para solicitar su intervención en la concesión de una vacante eclesiástica para sus hijos Juan y Pedro ${ }^{16} \mathrm{y}$, sabedor de la pasión artística del cardenal, le obsequia con numerosos retratos de la familia real. Estas peticiones no son nuevas para el cardenal, quien recibió múltiples cuadros del propio Tiziano con la esperanza de que el vicecanciller consiguiese un beneficio para su hijo Pomponio ${ }^{17}$.

La primera carta conocida es la que escribe el pintor al cardenal el 28 de abril de $1579^{18}$, junto a la que envía un retrato de Felipe II. En ella hace mención a los mandados al Papa Gregorio XIII «de su Magestad y de sus hijos». Ludovico Orsino, comendador de Bolonia, informa al cardenal Farnesio de este envío en una carta fechada en Madrid el 10 de mayo de 1579: "L'altro giorno io fui affanato da Alonso Sances pittor provisionato da sua $M^{\text {ta }}$ molto famoso in queste parti, a scrive a V.S.Ill ${ }^{\text {ma }}$ et $R^{m a}$ facendo fe de la devotione et osservanza che gli porta; et volsa $q^{\text {ta }}$ lettera da me per accompagnare un ritratto del Re molto naturale che porta in suo nome a donar a V.S.Ill ${ }^{\text {ma }}$ et $R^{m a}$ l'Abbate Floccazo che se ne torna a Roma; non ho potuto mancar a $q^{\text {to }}$ tanto virtuoso di $q^{\text {to }}$ offitio» ${ }^{19}$. R. M. Mulcahy supone que estas obras son réplicas del retrato del rey conservado en Pollok House (Glasgow) que fue pintado entre 1571-1572, y una vesión del retrato doble de los dos hermanos, Don Diego y Don Felipe, conservado en el convento de las Descalzas Reales y realizado en $1579^{20}$.

Estos regalos no son suficientes y Sánchez Coello envía nuevas cartas al cardenal durante el siguiente año, junto a un retrato de Don Sebastián de Portugal. En la primera de ellas, fechada en Madrid el 9 de abril de 1580 (Apéndice Documental, II), pide al cardenal que hable con el Da-

cinco o seis años y la técnica empleada. La variante inglesa está fechada en 1571 y, en ella, las efigiadas presentan una menor edad respecto al cuadro del museo madrileño, por lo que difícilmente puede considerarse una réplica como figura en el catálogo del Museo del Prado. Para I. S. Perlinguieri, en su estudio Sofonisba Anguissola. The Firts Great Woman Artist of the Renaissance, N. York, 1992, el cuadro inglés se debería al pincel de Sofonisba.

14 Sobre el cardenal Farnesio como mecenas artístico es fundamental el estudio de C. Robertson anteriormente citado.

15 R. M. Mulcahy, art. cit., 1992, supone que el nexo de unión sea el hermanastro, y también pintor, Jerónimo Sánchez que se encontraba en Roma en 1575. Sobre este personaje, ver el artículo de la mencionada estudiosa: «En la sombra de Alonso Sánchez Coello: la búsqueda por Jerónimo Sánchez», A.E.A., 250, 1990, pp. 304-309.

${ }^{16}$ La limitación de este artículo nos impide extendernos en las biografías de los dos hijos del pintor para las que sigue resultando imprescindible la obra de San Román, F. de B., «Alonso Sánchez Coello: Ilustraciones a su biografía», Boletín de la academia de bellas artes y ciencias históricas de Toledo, XII, 1930.

17 Ronchini, A., «Delle relazioni di Tiziano coi Farnese», Atti e Memorie delle R. Deputazioni di Storia Patria per le provincie modenensi e parmensi, 1864, pp. 129-146. Tampoco Sánchez Coello era nuevo en estas lides como demuestra su correspondencia con Granvela, quien escribe al cardenal Farnesio en 1585 alabando el arte del pintor y pidiendo que ayude a su hermanastro Jerónimo. Sobre esto último, véase Piot, C., «Alonso Coello, peintre espagnol à Bruxelles», Bulletin de l'Académie Royale des Sciences, des Lettres et des Beaux-Arts de Belgique, XXIX, 1895, pp. 304 y $310-311$.

18 Mulcahy, R. M., art. cit., 1992, Apéndice A. Todas estas cartas ya publicadas y las que damos a conocer se encuentran en el Archivo di Stato di Parma (A.S.Pr.). Aprovechamos esta nota para agradecer a su Director, Dott. Dall'Acqua, y a todo su personal, la calurosa acogida durante nuestra investigación en dicho archivo.

19 A.S.Pr., Carteggio Farnesiano, Estero, Spagna, b.128, fascículo año 1579. La necesidad de esta carta de presentación hace suponer que no había existido un contacto anterior entre ambos personajes. En el Archivo General de Simancas, Cámara de Castilla, libro 361, f. 137r., con fecha y de abril de 1579, se menciona que Scipion Flocari, capellán de S. M., va a Italia y lleva «tres retratos en lienço» que podrían identificarse con los que nos ocupan. Agradezco esta última noticia a la amabilidad de Miguel Morán Turina.

20 También se han identificado como los dos hermanos los dos niños que aparecen en un retrato de hacia 1580 reproducido en el cat. Alonso Sánchez..., 1989, p. 109, fig. 86. 
tario y que le avise si vaca algún beneficio eclesiástico para sus dos hijos estudiantes. Para inclinar la voluntad del cardenal, manda a través de Ludovico Orsino que va a Roma «el retrato del mal logrado rey de Portugal don Sebastian, aunque entiendo que le ha de hazer a V.S.Ill ${ }^{\text {ma }}$ gran lastima y dolor viendo Un Principe en tan floreciente edad y de tan grandes esperanças acavadas en un punto, el retrato es bien pareçido, y hecho 15 dias antes de la ynfelice jornada Dios le tenga ensu gloria». El 23 de julio de 1580, vuelve a escribir al cardenal para cerciorarse de que el retrato ha llegado a sus manos y suplicarle que interceda ante el Pontífice para que recaiga sobre su hijo Juan el beneficio, de quinientos ducados, vacante por la muerte, ese mismo mes, de Delgado, inquisidor de Córdoba ${ }^{21}$. El cardenal no hace oídos sordos a esta petición y escribe a Lodovico Bianchetti, el cual le responde el 28 de agosto comuniccándole que el beneficio no está vacante $^{22}$. Lo más interesante, desde un punto de vista artístico, de esta correspondencia de 1580 es el envío del retrato de D. Sebastián de Portugal realizado por Sánchez Coello. En la carta fechada el 9 de abril, el pintor explica que el retrato fue pintado quince días antes de que D. Sebastián falleciese en la batalla de Alcazarquivir el 4 de agosto de 1578. Sánchez Coello vino de Portugal a la corte castellana, en 1555, con un retrato de Don Sebastián casi recién nacido y realizó varias copias a lo largo de su carrera de los cuadros del portugués Cristóbal de Morales enviados desde Lisboa ${ }^{23}$. De creer las palabras del pintor, el retrato con que obsequió al cardenal representaría a Don Sebastián con veinticuatro años y posiblemente sería una copia ${ }^{24}$ más del último retrato del monarca luso enviado desde Portugal antes de la partida hacia Marruecos en junio de 1578. Los únicos retratos del hijo de la Princesa Juana que se podrían relacionar con éste son los derivados de un original perdido de Sánchez Coello que representaba al rey portugués con la orden militar de Cristo pendiendo de su cuello ${ }^{25}$. A nuestro entender, aunque este retrato se ha identificado con el prior de Crato D. Antonio, no resulta muy convincente desde un punto de vista histórico, que el pintor de cámara de Felipe II realice retratos de uno de sus adversarios en el trono portugués, ni tampoco que éstos se envíen al cardenal Farnesio, que no olvidemos, era tío abuelo de Ranuccio Farnesio, otro de los los aspiantes al trono luso al ser hijo de María de Portugal ${ }^{26}$. Aparte del matrimonio entre la mencionada princesa y Alejandro Farnesio en 1565, el cardenal mantuvo una estrecha relación con Portugal desde su nombramiento como obispo de Viseo en 1552 y su papel como cardenal protector de Portugal en Roma. Estos vínculos explicarían la elección de este retrato para ser enviado al cardenal Farnesio.

${ }^{21}$ Mulcahy, R. M., art. cit., 1992, p. 308. Hemos localizado una copia de esta carta, con ligeras variantes ortográficas, en el fondo Carteggio Farnesiano, Estero, Spagna, b.128.

${ }^{22}$ Robertson, C., op. cit., 1992, p. 309, n. ${ }^{\circ} 110$, Esta carta está fechada en Roma el 29 de agosto de 1580 y se conserva en el A.S.Pr., Carteggio Farnesiano, Estero, Roma, b.388.

${ }^{23}$ Sobre los retratos del rey Don Sebastián resulta de gran interés el estudio de Jordan, A. M., Retrato de corte em Portugal, o legado de António Moro (1552-1572), Lisboa, 1994. En especial, se analiza esta cuestión en las pp. 116-127 y en el catálogo final de los n. ${ }^{\text {os }} 58$ a 78 .

${ }^{24}$ No existe ninguna constancia de que Sánchez Coello visitase la corte portuguesa en 1578, por lo que no podría haber sacado este retrato de D. Sebastián del natural. Posiblemente, copiase algún retrato enviado a la corte española desde Lisboa.

${ }^{25}$ Se conservan varias copias de este retrato de Sánchez Coello fechable hacia 1578: en el Kunsthistorisches Museum de Innsbruck ( $n .^{\circ}$ inv 3282), Kunsthistorisches Museum de Viena (n. ${ }^{\circ}$ inv. 3493), Museo del Prado (n. ${ }^{\circ}$ inv. 5764, en depósito en el Museo de S. Telmo de S. Sebastián). A. M. Jordan, al analizar este grupo de retratos, manifiesta sus dudas de que el efigiado sea D. Sebastián y cree más probable que se trate de D. Antonio, prior de Crato. En el libro de la mencionada autora, no se alude a una copia de un retrato, cuya inscripción identifica con D. Sebastián, conservado en el convento de las Descalzas Reales, muy similar al que creemos que fue enviado al cardenal Farnesio, en el que Sebastián aparece armado como en el grabado de Perret (Lafuente Ferrari, E., Iconografía Lusitana. Retratos grabados de personajes portugueses, Madrid, 1941, n. ${ }^{\circ}$ 500). Por su parte, C. Robertson, op. cit., 1992, p. 146, sugiere que la miniatura conservada en la Galleria Nazionale de Parma (n. ${ }^{\circ}$ inv. 1177/13) deriva del retrato enviado por Sánchez Coello al cardenal. Esta hipótesis es duramente debatida por A. M. Jordan, op. cit., 1994, p. 172, cat. 70, que estima que este retrato debe identificarse con D. Antonio.

${ }^{26}$ Esta princesa era hija de D. Duarte y, por lo tanto, nieta del rey portugués Manuel I. 
Con fecha del 7 y 23 de enero de 1581, llegaron dos nuevas cartas de Sánchez Coello al cardenal en las que el pintor se pone a su servicio y solicita alguna merced para sus hijos ${ }^{27}$. Es en la siguiente carta, fechada el 18 de noviembre de $1582^{28}$, donde volvemos a encontrar, además de los consabidos ruegos para que favorezca a sus hijos, nuevos envíos de retratos. En esta ocasión, el pintor comunica a Farnesio que cuando recibió su última carta, hacía alrededor de un mes que había enviado dos retratos del príncipe Don Diego ${ }^{29}$, uno para Farnesio y otro para el Papa, y le ruego que le haga saber si desea algún otro retrato en particular. El cardenal hace acuso de recibo en una carta enviada al pintor el 23 de mayo de 1583 a la que Sánchez Coello responde el 4 de julio ${ }^{30}$ haciéndole saber que el príncipe heredero ha fallecido y que su hijo Juan conseguirá el año próximo el título de licenciado.

Los siguientes indicios de correspondencia epistolar datan del año 1585. A raíz del envío de un nuevo cuadro de Felipe II $^{31}$, el 21 de julio, Alessandro Farnesio escribe una carta al Datario, que portará el propio Juan Sánchez Coello, que se encontraba ya en Roma. El cardenal, aparte de solicitar un beneficio para el joven, se declara muy amigo del pintor cuyo arte era muy admirado por el Papa Gregorio XIII ${ }^{32}$. Ocho días después, el cardenal escribe desde Caprarola al pintor agradeciéndole el retrato real y comunicándole que ha conocido a su hijo Juan, ya en Roma, a quien intentará ayudar en todo lo posible ${ }^{33}$.

El pintor vuelve a escribir al cardenal el 4 de mayo de 1587 (Apéndice Documental, III). En esta carta anuncia que contestó a la enviada por Farnesio el 24 de marzo y, en un cierto tono de reproche, le recuerda sus promesas incumplidas de conseguir una vacante para el joven que ya lleva casi dos años en Roma, lo que resulta enormemente oneroso al pintor ${ }^{34}$.

${ }^{27}$ Mulcahy, R. M., art. cit., 1992, p. 308.

28 Ibidem.

${ }^{29}$ Es difícil determinar de qué retrato se trata, pues el único retrato firmado, en 1577, que se conserva está en una colección privada inglesa y proviene de la colección real española. Sin embargo, S. Breuer-Hermann en su estudio «Alonso Sánchez Coello vida y obra», Cat. Alonso Sánchez Coello..., Madrid, 1990, pp. 27-28, da a conocer el encargo, por parte del Rey, de un retrato de Don Diego armado para el Emperador de la China y de una cuenta conservada en el Archivo de Palacio de Madrid, se deduce que el 20 de abril de 1582, Sánchez Coello había pintado un retrato del príncipe vestido con un traje de raso rojo bordado con calzas y guerrera. Este retrato fue enviado, por orden del Rey, a Flandes donde todavía se encontraban Margarita de Austria y su hijo Alejandro Farnesio. Resulta tentador relacionar con estos dos últimos retratos perdidos aquéllos enviados a Roma.

${ }^{30}$ Mulcahy, R. M., art. cit., 1992, p. 308.

${ }^{31}$ El único retrato de Felipe II de la colección Farnesio que ha llegado a nuestros días, es el de Tiziano conservado en la Galleria Nazionale de Capodimonte (n. ${ }^{\circ}$ inv. Q127) y que fue utilizado por Zuccaro en los frescos de Caprarola como sugiere Wethey, H. E., The Paintings of Titian. Complete Edition. Volume II. The Portraits, Londres, 1971, pp. 128-129, n. ${ }^{\circ} 79$, que lo identifica con uno citado en 1626 y 1651 en la villa. Al ir estas obras destinadas al cardenal Farnesio, resulta lógico que se conservasen o en el palacio romano o en Caprarola. El primer inventario que se conserva del primero data de 1644 y ha sido publicado por Jestaz, B., L'inventaire du Palais et des propiétés Farnèse à Rome en 1644, Roma, 1994. En él encontramos algunos retratos del Rey que podrían ser los que nos ocupan: p. 59, n. ${ }^{\circ}$ 996: «Uno [quadro] in tela grande al naturale con il ritratto di Filippo $2^{\circ}$ con cornice bianca» que se aproximaría al de Tiziano; p. 92, n. ${ }^{\circ} 2071$ : «Uno in tela di Filippo $2^{\circ}$, senza cornice», que en el inv. de 1653 (la parte de las pinturas ha sido publicada y estudiada por Bertini, G., La Galleria del Duca di Parma. Storia di una collezione, Bolonia, 1987, p. 208, n. ${ }^{\circ}$ 52) se precisaría que va armado y porta el Toisón, lo que le acercaría al de Glasgow. El primer inventario de Caprarola data de 1681 y se conserva en el A.S.Pr., Casa e Corte Farnesiana, serie VIII, b.52, fasc. 6. En el Camerino della Torre, junto a un retrato de Carlos V, encontramos «Il ritratto di filippo secondo simile senza cornice» (f. 6v) o en la Stanza dello Studio, junto a estatuas, bronces, etc. "un quadro grande di filippo $2^{\circ " \prime}$ (f. 20).

32 Robertson, C., op. cit., 1992, p. 311, n. ${ }^{0} 123$.

${ }^{33}$ Mulcahy, R. M., art. cit., 1992, p. 308. La palabra que aparece como ilegible es «tiene». Este nuevo envío consigue que el cardenal reaccione positivamente a la petición de Granvela en favor del hermano de Sánchez Coello como demuestran las cartas dadas a conocer por Piot, C., art. cit., 1895.

${ }^{34}$ De esta carta se deduce que la primera idea de Sánchez Coello fue que su hijo residiese en el propio palacio romano que acogió a numerosos artistas y eruditos vinculados con la corte española como se refleja en el estudio de Vegué y Goldoni, A., «En torno a la figura del Greco», Arte Español, 1926, t. VIII, pp. 70-81. Asimismo, esta carta resulta de enorme interés desde un punto de vista sociológico al informarnos de la situación económica del artista. 
El año siguiente, último de su vida, Sánchez Coello vuelve a recurrir al envío de un retrato del entonces príncipe heredero Don Felipe ${ }^{35}$. Farnesio le contesta el 22 de febrero agradiciéndole el cuadro, lo que da pie a una nueva carta del pintor el 1 de abril (Apéndice Documental, IV) en la que muestra su satisfacción porque el retrato haya gustado al cardenal, "no por ser de mi mano sino por el amor y voluntad que V.S.Ill. tiene a su magd y a sus cosas» —afirmación con la que no estaría muy de acuerdo el cardenal que fue apartado, una y otra vez, del trono pontificio por el veto del monarca - a la vez que se pone a su disposición si el cardenal desea alguna pintura suya. Sánchez Coello no olvida recordar el asunto pendiente de su hijo para el que solicita un beneficio con el que pueda sustentar a sus cuatro hermanas doncellas. La última carta enviada por el pintor el 28 de mayo de 1588 (Apéndice Documental, V) insiste sobre el argumento anterior.

Desgraciadamente, no sabemos si todos estos desvelos de Sánchez Coello contribuyeron a que su hijo Juan consiguiese la plaza de capellán de la Capilla de los Reyes Nuevos de Toledo en 1593 y si los oficios del cardenal Farnesio, fallecido en 1589, tuvieron algo que en ello. La importancia de esta correspondencia reside en que nos aproxima tanto al pintor del monarca más poderoso del momento, como a uno de los mecenas de mayor relevancia de la Roma de la épo$\mathrm{ca}$, a la vez que contribuye a arrojar luz sobre obras poco conocidas del primero y sobre las que formaban la fabulosa coleccioón del segundo.

AlMUdena PÉREZ DE TUdela Becaria Universidad Complutense

\section{APÉNDICE DOCUMENTAL \\ (Correspondencia entre Alonso Sánchez Coello y la familia Farnesio)}

I. Carta de Sebastián de Santoyo a destinatario desconocido, San Lorenzo de El Escorial, 9 de marzo de 1572. (Archivio di Stato di Parma (A. S. Pr.), Epistolario Scelto, busta 19 (Pittori)).

señor / La de V. md. de siete deste Reçibi: oy domingo por la mañana. que se quentan nueve, y por la horden que V. md. me escribe. que el señor $\mathrm{p}^{\circ}$ aldobrin [Aldobrandini] trae de madama de llevarle los retratos, de las señoras. ynfantas pregunte a su mag ${ }^{\mathrm{d} \text {. }}$ si daria liçençia para que $\mathrm{al}^{\mathrm{o}}$ sanchez entraste a rretratar: a sus altezas, dize que holgara el dello. pero por estar: la mayor dellas tan descolorida y malita como esta no le pareçe ser este tiempo a proposito, que llegado ay. y aviendo mejorado la niña se lo torne a acordar, que el dira. La forma que se terna para averlas de rretratar. y ymbiar los etratos a la duquessa aqui se entiende que la buelta a esse pueblo sera por toda esta semana y con tanto no terne mas que deçir sino que nro $\mathrm{s}^{\mathrm{r}}$ guarde $\mathrm{y}$ acreçiente. La persona y casa de V. md. como desea de sant lorenço El rreal a nueve de Março de MDLXXII anos. / B.l.m.a.v.m. su rey. / Sebastian de santoyo.

II. Alonso Sánchez Coello al cardenal Alessandro Farnesio, Madrid, 9 de abril de 1580. (A.S.Pr., Carteggio Farnesiano, Estero, Spagna, b.128).

${ }^{35}$ El único retrato individual del futuro Felipe III pintado por Sánchez Coello, es el conservado en el Museo de San Diego de California. En el inventario del Palacio Farnesio de Roma de 1653 (A.S.Pr., Raccolta Manoscritti, b.86, f. 228), aparece «un retrattino [posiblemente una miniatural] di filippo Pnpe di Spagna nell'età d'anni 9 cornicetta d'ebbano». En el inventario del mismo palacio de 1644 (Jestaz, B., op. cit., 1994, p. 91, n. ${ }^{\circ}$ 2052), encontramos: «Un quadretto in tela del re Filippo $2^{\circ}$ in età puerile con cornice di legno», que en el inventario de 1653 (Bertini, G., op. cit., p. 208, n. ${ }^{\circ}$ 47) aparece como: «Un quadro in tela col ritratto di Filippo $2^{\circ}$ giovine con un ucello in pugno cornice di noce». Este retrato podría ser uno de los infantes confundido, andando el tiempo, con el del progenitor. En el inv. del Palacio Farnesio de 1644 n. ${ }^{\circ} 998$ aparece: «Uno [quadro] simile [al n. ${ }^{\circ} 996$ de Felipe II] con il ritratto d'un Infante figliolo del re di Spagna», que se podría identificar con alguno de los enviados por Sánchez Coello. 
III $^{\text {mo }}$ señor / Aunque ha pocos dias que escrevi a V.S.III ${ }^{\text {ma }}$ en respuesta de dos cartas que V.S.II ${ }^{\text {ma }}$ me hizo merced de escrevirme. No he querido agora siendo el portador el señor Ludovico Orsino dexar de recivirla de Nuevo tornando a escrevir a V.S.Ill. a quien supp ${ }^{\text {co }}$ humilm $^{\text {te }}$ se acuerde que tiene aqui V.S. un afficionadissimo criado y servidor para todo Lo que V.S. Ill. me quisiere mandar que yo como tal no pienso (en cualquier occassion que se me iffrezca) dexar de ymportunar a V.S.IIl ${ }^{\text {ma }}$ me haga mrd., y assi en la que V.S. me ha hecho y haze intercediendo con su $\mathrm{S}^{\mathrm{d}}$ me haga alguna merced para dos hijos estudiantes que tengo, para que mejor puedan passar adelante con sus estudios, creo yo muy bien que ha sido con La Voluntad que V.S. me escrive, y para que la mrd. sea del todo cumplida, supp ${ }^{c o}$ a V.S. Ill. que porque el avisar yo de alguna Vacante podria ser negocio muy largo, assi porque quando se offece alguna, es menester despachar a mucha costa correo propio, y yo tengo poca possibilidad para ello, o porque también podría no offrecerseme ocasion en muchos dias, me haga V.S.Ill ${ }^{\mathrm{ma}}$ tanta mrd. de hablar al Datario para que avise a V.S. de alguna que pueda hazerme su $S^{d}$ mrd. por intercession de V.S.Ill ${ }^{\text {ma }}$, que padres y hijos, reconoceremos toda la Vida la obligacion tan grande en que V.S. nos ha puesto con tanta mrd. como nos haze. / Ay embio a V.S. el retrato del mallogrado rey de Portugal don Sebastian, aunque entiendo que le ha de hazer a V.S.Ill ${ }^{\mathrm{ma}}$ gran lastima y dolor Viendo Un Principe en tan floreciente edad y de tan grandes esperanças acavadas en Un punto, el retrato es bien pareçido, y hecho 15 dias antes de la ynfelice jornada Dios le tenga en su gloria y guarde la Ill $^{\text {ma }}$ persona de V.S. y en mayor estado acreciente en su sancto servi ${ }^{\circ}$, como los criados de V.S.Ill ${ }^{\text {ma }}$ deseamos, de Madrid 9 de abril 1580. / Ill $^{\text {mo }} \mathrm{S}^{\text {or }} /$ Bessa las Ill ${ }^{\text {mas }}$ manos de V.S. / su criado y servidor / $\mathrm{A}^{\circ}$ Sanchez Coello.

III. Carta de Alonso Sánchez Coello al cardenal Farnesio, Madrid, 4 de mayo de 1587. (A.S.Pr., Carteggio Farnesiano, Estero, Spagna, b.129).

IIl $^{\text {mo }}$ señor / Aunque con el ordinario escrevi a V.S.Ill ${ }^{\text {ma }}$ en respuesta de su carta de XXIII' ${ }^{\circ}$ de Março, porque no estoy cierto si La avra V.S.Ill ${ }^{\text {ma }}$ recevido, no puedo dexar de hazerlo agora, y todas las vezes que se offreciere ocasion, y supplicar a V.S.Ill ${ }^{\mathrm{ma}}$, como Lo hago, considere que mi intención quando embie a esa corte a mi hijo fue con la confiança que tuve de la mrd que V.S.Ill ${ }^{\text {ma }}$ me avia offecido por sus cartas. Y entendiendo que V.S.Ill ${ }^{\text {ma }}$ fuera servido de recibirle en su casa como a los demas criados y que quando en esto no tuviera Ventura no le faltara en aver salido con alguna Vacante con el favor y amparo de V.S.IIl ${ }^{\text {ma }}$, en cerca de dos años que ha que asiste ay. porque de otra manera yo no me atreviera ni tengo posibilidad para sustentarle, porque certifico a V.S.Ill ${ }^{\text {ma }}$ que me cuesta ya mas de seiscientos ducados, y que en ninguna manera si V.S. no Lo remedia con tomar esta causa con las Veras que confio le puedo sustentar mas en esa corte ni tampoco despachar correo propio quando alguna cosa vaca: y pues agora los ss. Cardenales tienen Los indultos de las vacantes supp $^{\text {co }}$ a V.S.IIl ${ }^{\text {ma }}$ Les pida alguna que haziendome V.S.Ill ${ }^{\text {ma }}$ esta mrd y pudiendo V.S. tanto con todo el mundo quedo confiado que tendra todo buen successo, el me escrive La mrd que V.S.Ill. le haze en todo y La que espera que le hara en sus pretensiones de lo qual estoy yo muy confiado y tambien lo estoy de que V.S.Ill. perdonara esta pesadumbre por ser tocante a hijo y de tan criado de V.S.Ill. Cuya Ill ${ }^{\text {ma }}$ persona N.S. ge y su gran estado acreciente. De Madrid. 4. de Mayo 1587. / $\mathrm{Ill}^{\text {mo }} \mathrm{S}^{\text {or }} /$ B.l.m.de.V.S. Ill ${ }^{\text {ma }} / \mathrm{su}$ menor criado / $\mathrm{A}^{\circ}$ Sanchez Coello.

IV. Carta de Alonso Sánchez Coello al cardenal Farnesio, Madrid, 1 de abril de 1588. (A.S.Pr., Carteggio Farnesiano, Estero, Spagna, b.129).

$\mathrm{Ill}^{\mathrm{mo}} \mathrm{s}^{\text {or }}$ / Con la de V.S.Ill de 22 de Hebrero recibi grandissima mrd como sera todas Las vezes que v.s.Ill. me la hiziere y me quisiere mandar en que le sirva, de que el retrato del Principe $N S^{\text {or }}$ aya dado a V.S.Ill. contento estava yo bien cierto no por ser de mi mano sino por el amor y voluntad que V.S.Ill. tiene a su mag ${ }^{d}$ y a sus cosas, harta mrd seria para mi que V.S.Ill. me ocupasse en su servi ${ }^{\circ}$ en cosas de mi arte pues nadie en el mundo Lo hara con mas Voluntad que yo, de La que V.S.Ill. haze al licenciado Juan Sanchez Coello mi hijo estava yo confiado y assi espero que con mucha brevedad V.S. Ill. le han de colocar en algun buen lugar para que pueda vivir honrradamente y favorecer a quatro hermanas donzellas que tiene y quitarme a mi de costa que cierto con esperança de la mrd. que V.S.Ill. le ha de hazer he sacado fuerças donde no las hay para que asista ay y assi La tengo agora de que por intercesion de V.S.Ill. Le ha de succeder como todos desseamos, N.S ${ }^{r}$ guarde la Ill. pers ${ }^{\mathrm{a}}$ de V.S. y su gran estado y dignidad acreciente como desseo. De Madrid prim ${ }^{\circ}$ de abril 1588. / Ill ${ }^{\mathrm{mo}} \mathrm{S}^{\text {or }} /$ B.l.m.a V.S. Ill ${ }^{\mathrm{ma}}$ / su criado y servidor / $\mathrm{A}^{\mathrm{o}}$ Sanchez Coello. 
V. Alonso Sánchez Coello al cardenal Farnesio, Madrid, 28 de mayo de 1588. (A.S.Pr., Carteggio Farnesiano, Estero, Spagna, b.129).

$\mathrm{Ill}^{\mathrm{mo}} \mathrm{S}^{\text {or } ~ / ~ N o ~ p u e d o ~ d e x a r ~ d e ~ i m p o r t u n a r ~ a ~ v . s . I l l . ~ l a s ~ v e z e s ~ q u e ~ s e ~ o f f r e c i e r e ~ o c c a s i o n ~ y ~ t r a e r ~ a ~ l a ~ m e m o-~}$ ria al licenciado Juan Sánchez mi hijo, para que v.s.Ill ${ }^{\mathrm{ma}}$ La tenga de hazerle merced en alguna vacante, pues agora ay mejor ocasion de poderlo hazer por aver tomado su $\mathrm{s}^{\mathrm{d}}$ en si los indultos segun aca se dize que su s $\mathrm{s}^{\mathrm{d}}$ avia dado a los ss. cardenales: porque Le certifico a V. S. Ill. que en el tiempo que ha que reside en esa corte que son tres años, como esta a mi costa me ha gastado mucha hazienda, y me la gasta, que me ha puesto y pone en no pequeña necessidad, si v.s.Ill. no lo remedia como me lo tiene offrecido tantas vezes, yo no se que me haga sino es dar gracias a N.S. que ha sido servido y Lo deve ser desto, por lo cual supp ${ }^{\text {co }}$ a V. S.Ill, que pues Le tomo debaxo de su amparo y protección, y el por su persona no desmerece qualquiera mrd que se le haga: ni yo por mi voluntad y desseo de acertar a servir a V.s.Ill. tampoco, favorezca su causa de manera que salga cierta mi esperança y el con alguna cosa para que del todo yo no me pierda por su occasion, y pueda favorecer quatro hermanas donzellas que tiene, porque sentiría mucho que sabiendo aqui todos La mrd que V.s.Ill. haze a mi hijo, viessen despues que avia sido desfavorecido, pues pudiendolo V.s.Ill. todo el se quedava sin nada que segun son las gentes juzgarían que de meritos suyos devia ser la causa desto, yo quedo confiado que V.S.Ill, remediara estos daños con hazer de manera que se le de alguna vacante: si para alguna cosa del servi ${ }^{\circ}$ de v.s.Ill. fuere yo menester no tengo que offrecerme de nuevo quien ha tantos dias que Lo esta sino que V.S.Ill. me emplee en el pues sera para mi tanta mrd, N.S ${ }^{\mathrm{r}} \mathrm{g}^{\mathrm{e}}$ y acreciente La Ill $^{\mathrm{ma}}$ persona y gran estado

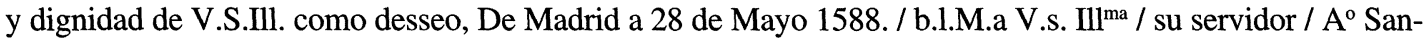
chez Coello.

\section{UN RETRATO DE JUAN DE BRIVIESCA POR JERÓNIMO LÓPEZ POLANCO}

Desde que el actual palacio episcopal de Segovia fuera adquirido en el siglo XVIII para servir de residencia a los obispos de la diócesis, el edificio de la plaza de San Esteban ha ido acumulando en su interior un variado, en cuanto a calidad y estilo, conjunto de objetos y obras de arte que han formado una valiosa y singular colección. La compra de obras con el fin de decorar las estancias del palacio, la desamortización eclesiástica del siglo XIX, el intento de crear un museo diocesano en 1921 o el despoblamiento del medio rural durante el siglo XX son algunos de los hechos que han contribuido a formar esta colección.

Aunque el palacio siempre tuvo un acceso restringido, muchas de sus mejores pinturas y esculturas han sido ya estudiadas y publicadas; otras, desconocidas, pero de indudable mérito, esperan aún el momento oportuno para salir a la luz. Gracias a la creación del Museo «Palacio Episcopal», inaugurado en junio de 1995, se ha conseguido no sólo la restauración y catalogación de una significativa parte de sus fondos, sino también la difusión y puesta en valor de los mismos.

El lienzo objeto de estas líneas es un retrato del sacerdote y terciario franciscano Juan de Briviesca, realizado por el pintor madrileño Jerónimo López Polanco, obra localizada en el palacio desde fecha desconocida y «rescatada» del lugar donde se encontraba almacenada para ser expuesta, tras su oportuna restauración, en una de las salas del museo (Figs. 11 y 12) ${ }^{1}$. En los es-

\footnotetext{
${ }^{1}$ La restauración, llevada a cabo en Junio de 1995, consistió en un forrado de la debilitada tela original, una sustitución del bastidor y un sentado y limpieza de la capa pictórica. Al carecer de marco, se adaptó uno de época que, sin lienzo, se conservaba en el Museo.
} 